\title{
Intranasal supernumerary tooth in a child: a case report
}

\author{
Yoon Seok $\mathrm{Choi}^{1 \oplus}$, Yong-Dae Kim ${ }^{1,2}$, Chang Hoon Bae ${ }^{1 \oplus}$, Hyung Gyun $\mathrm{Na}^{1 \oplus}$ \\ ${ }^{1}$ Department of Otorhinolaryngology-Head and Neck Surgery, College of Medicine, Yeungnam University, Daegu, Republic of Korea; \\ ${ }^{2}$ Regional Center for Respiratory Diseases, Yeungnam University Medical Center, Daegu, Republic of Korea.
}

\begin{abstract}
Background. Ectopic eruption of supernumerary teeth in the nasal cavity is extremely rare, and most cases usually involve the maxillary sinus or are accompanied by dental cysts. It is usually discovered during adulthood.

Case. A 5-year-old patient presented with an intranasal mass and intermittent nasal bleeding that lasted for 1 year. He was taking medication for symptoms of allergic rhinitis, such as nasal obstruction and intermittent epistaxis, without any endoscopic evaluation for 1 year. On nasal endoscopy, a needlelike whitish mass was observed on the left nasal floor. On paranasal sinus computed tomography, it appeared as a pointed highdensity mass covered by soft tissue. The intranasal mass which was a supernumerary tooth was completely removed using a pediatric endoscope.

Conclusions. Detection of supernumerary teeth in the nasal cavity of children without symptoms is difficult, and it can be delayed; although the child, in this case, had nonspecific nasal symptoms, supernumerary teeth was not considered in the diagnosis. This case report raises awareness and provides evidence for the clinical characterization and optimal treatment of supernumerary teeth in children.
\end{abstract}

Key words: supernumerary tooth, nasal cavity, child, nasal obstruction, epistaxis.

Supernumerary teeth refer to deciduous and permanent teeth that occur in addition to the normal number of teeth; the prevalence of supernumerary teeth is approximately 0.1 $1 \%$ of the general population. The biological and genetic mechanisms underlying supernumerary nasal teeth remain largely unclear. Supernumerary teeth are generally characterized by a specific morphology. The most common form is the conical type, with the tuberculate or barrel-shaped and molariform types occurring less frequently. ${ }^{1}$

Ectopic eruption of supernumerary teeth in the nasal cavity is extremely rare, and most cases usually involve the maxillary sinus or are accompanied by dental cysts. In the oral cavity, the most common supernumerary tooth

\footnotetext{
$\triangle$ Hyung Gyun Na drnhk79@gmail.com
}

Received 13th May 2020, revised 25th June 2020, accepted 16th December 2020. is the mesiodens, which is found between the upper central incisors. Other rare locations of supernumerary teeth include the mandibular condyle, coronoid process, orbit, and nasal cavity. ${ }^{2}$ Although most cases are asymptomatic, supernumerary teeth in the nasal cavity have been reported to cause recurrent nasal bleeding, facial pain, headache, nasal septal abscess, and oroantral fistula. ${ }^{3}$ It may also impact normal tooth eruption and lead to the development of malocclusion in children.

Most cases are discovered during adulthood, with most patients showing rhinologic symptoms. ${ }^{4}$ However, we report the case of a 5-year-old child with ectopic eruption of a tooth on the nasal floor, which was characterized by nonspecific symptoms.

\section{Case Report}

A 5-year-old boy presented to the hospital with an accidentally discovered intranasal mass 
and recurrent nasal bleeding. The patient had allergic rhinitis and was under medication. Both his parents were also under medication for allergic rhinitis. On anterior rhinoscopy and nasal endoscopy, a sharp-pointed white mass enclosed in mucous membrane was discovered on the left nasal floor (Fig. 1). The mass occupied the nasal floor, and its sharp tip protruded toward the inferior turbinate. Other nasal findings were normal.

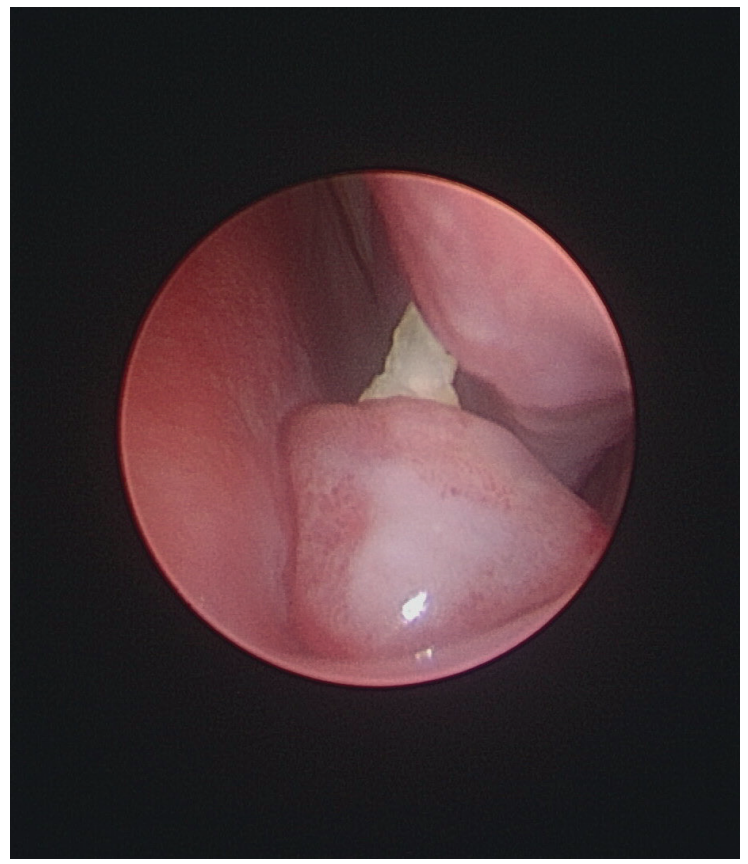

Fig. 1. Endoscopic finding, showing sharp-pointed white mass enclosed in a mucous membrane discovered on the left nasal floor.
Computed tomography (CT) of the paranasal sinus showed a pointed, high-density mass covered by soft tissue; there were no other significant findings (Fig. 2).

The mass was surgically removed under general anesthesia. It was located $3 \mathrm{~cm}$ posterior to the nostril and was firmly embedded in the floor of the nasal cavity. We initiated surgery by injecting $2 \%$ xylocaine and 1:100,000 adrenaline near the mass. Subsequently, we attempted to extract the mass with dental forceps, but we could not insert it due to the young age of the patient and the small size of the nostrils. Eventually, a complete resection involving the surrounding mucous membrane was performed using a Bovie needle while observing the mass with a pediatric endoscope. There was no osteoclasia of the surrounding tissues or other malignant findings. The excised mass was approximately $10 \mathrm{~mm}$ in length, with the crown facing the nasal cavity; the root structure was not clear (Fig. 3). The defect in the left nasal cavity resulting from the excision of the mass was not sutured. Subsequently, a nasal packing material was inserted into the defect, and pressure was applied to control bleeding. No abnormal findings were observed after surgery, and the patient was discharged on postoperative day 2 . The nasal congestion and bleeding resolved after excision, and no abnormal findings were observed during the one-year follow-up assessments.
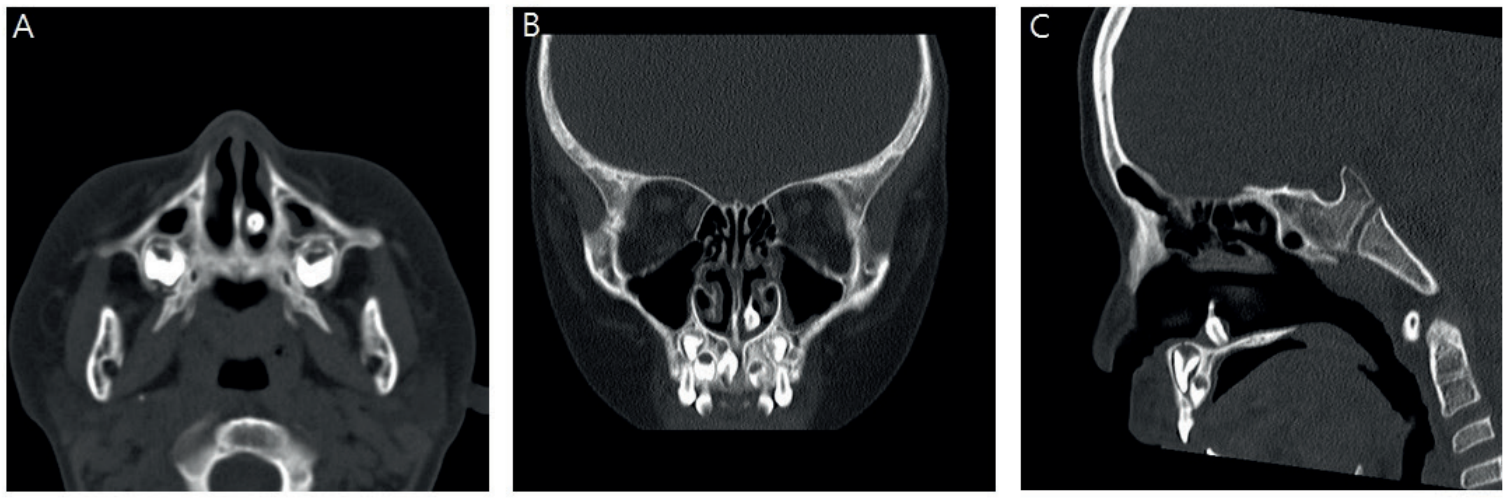

Fig. 2. Computed tomography (CT) of paranasal sinuses: Tooth observed in left nasal cavity floor. A. axial view, B. coronal view, C. sagittal view. 


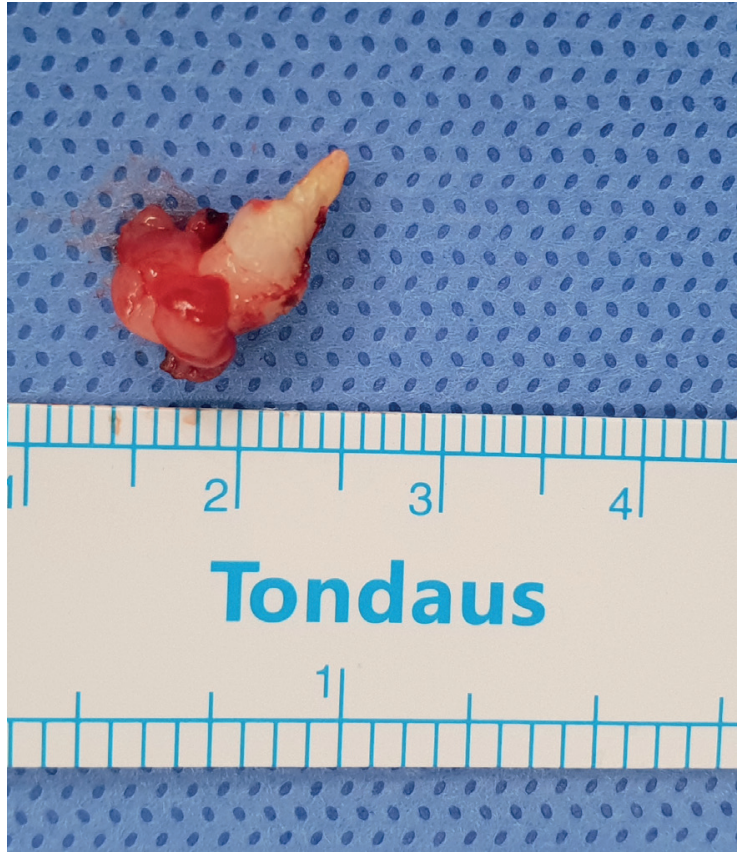

Fig.3.Supernumerary tooth, showing supernumerary tooth was completely removed.

This study was approved by the Institutional Review Board for Human Studies of the Yeungnam University Medical Center, Daegu, Korea. Permission was obtained from the child's parents for the publication of this case, and informed consent was obtained from the family.

\section{Discussion}

The etiology of supernumerary teeth is unknown; however, possible causes include an obstruction at the time of tooth eruption due to crowded dentition, persistent deciduous teeth, and developmental disturbances such as cleft palate, infection, trauma, and cysts. ${ }^{5}$ Ectopic teeth in the nasal cavity, as observed in this case, usually occur during tooth development, when the dental sac in the maxilla is inserted into the nasal cavity before palatal closure. Supernumerary teeth in the nasal cavity often present as a firm, fixed, and whitish mass that is often enclosed in granulation or necrotic tissue.

Radiologic testing may be useful for diagnosis, and the presence of a supernumerary tooth can be identified from the shadows observed in Waters' or Caldwell's views. However, in our patient, a complete radiographic series was not performed as the diagnosis was made on endoscopic physical examination and CT. CT may be used as an additional diagnostic tool for such cases. In most cases, the supernumerary tooth is found as a mass with a shadow that has a similar density to the teeth and bone. ${ }^{6}$ The tooth may also show permeability, suggesting the central location of the pulp cavity within the tooth. In addition to the confirmation of ectopic tooth growth, CT imaging may be a useful diagnostic tool to determine the presence of dental cysts, examine the relationship with surrounding tissues, and detect hidden ectopic tooth growth. For pediatric patients, in particular, the paranasal sinus $\mathrm{CT}$ should not be used for routine diagnosis; however, it may be used in cases of suspected tumors, application from sinusitis, and before sinus surgery to confirm the surgical anatomy. As observed in this case, a supernumerary tooth in the nasal cavity should be differentiated from a foreign body, rhinolith, benign or malignant mass, tuberculosis, mycotic infection, and osteoma in the nasal cavity.

The most common cause of nasal congestion in children is inflammation associated with conditions such as the common cold, paranasal sinusitis, and allergic rhinitis ${ }^{7}$; hence, the diagnosis of supernumerary teeth is often delayed, and the symptoms are neglected. However, when nasal symptoms are present, misdiagnosis can be prevented by suspecting a congenital or iatrogenic mass; a systematic approach involving history taking and physical examination should also be employed. The most effective method to diagnose sinonasal problems is through direct examination with a flexible endoscope. When a patient complains of symptoms, direct examination with an endoscope should be prioritized. Prompt treatment is recommended to prevent complications and structural changes. As observed in our patient, ectopic tooth eruption in children is often accidentally discovered during 
endoscopy or CT. Diagnosis is also delayed because patients are often asymptomatic. In this case, the child's family members had severe allergic rhinitis; thus, nasal congestion was attributed to this condition, and the child continuously took medications for allergic rhinitis. However, he visited our institution due to worsening nasal congestion and bleeding, and was subsequently diagnosed on visual inspection. As demonstrated in this case, visual inspection and diagnostic evaluation are important when nasal congestion is present in children.

Ectopic teeth in children are removed to alleviate symptoms and prevent complications such as interference with normal tooth eruption. However, nonerupted teeth without symptoms may be left untreated and periodically observed for progression. Owing to advances in endoscopy, these teeth can be removed with minimal injury to the surrounding tissues or mucosa. Furthermore, ectopic teeth within the maxillary sinus can be easily removed by securing a good field of view with the CaldwellLuc procedure. In this case, the patient was symptomatic, but with episodes of intermittent bleeding as the tooth had erupted in the nasal floor. We completely removed the tooth using nasal endoscopy, ensuring minimal injury to the surrounding tissue.

As in the reported case, the differential diagnoses of ectopic teeth include foreign bodies, exostoses, and odontomes. Calcifying odontogenic cysts or malignant tumors such as chondrosarcomas and osteosarcomas are also relevant differential diagnoses as they are associated with several nonspecific nasal symptoms. ${ }^{8,9}$ In addition, nasal endoscopic evaluation is essential for patients with rhinologic symptoms that are not sufficiently controlled by medical treatment. During physical examination, surgeons and physicians should rule out pathologies using flexible endoscopy, even in patients with a history of allergic rhinitis and chronic rhinitis.

\section{Acknowledgement}

This work was supported by the 2018 Yeungnam University Research Grant.

\section{Author contribution}

The authors confirm contribution to the paper as follows: study conception and design: NHG, CYS; data collection: $\mathrm{BCH}$; analysis and interpretation of results: KYD, BCH, NHG; draft manuscript preparation: NHG. All authors reviewed the results and approved the final version of the manuscript.

\section{Conflict of interest}

The authors report no conflicts of interest. The authors alone are responsible for the content and writing of this article.

\section{REFERENCES}

1. Ogane S, Watanabe A, Takano N, Shibahara T. Case of inverted supernumerary tooth in nasal cavity. Bull Tokyo Dent Coll 2017; 58: 255-258.

2. Lee JH. A nasal tooth associated with septal perforation: a rare occurrence. Eur Arch Otorhinolaryngol 2006; 263: 1055-1056.

3. Lin IH, Hwang CF, Su CY, Kao YF, Peng JP. Intranasal tooth: report of three cases. Chang Gung Med J 2004; 27: 385-389.

4. Kirmeier R, Truschnegg A, Payer M, Malyk J, Daghighi S, Jakse N. The supernumerary nasal tooth. Int J Oral Maxillofac Surg 2009; 38: 1219-1222.

5. Rajab LD, Hamdan MA. Supernumerary teeth: review of the literature and a survey of 152 cases. Int J Paediatr Dent 2002; 12: 244-254.

6. Chen A, Huang JK, Cheng SJ, Sheu CY. Nasal teeth: report of three cases. AJNR Am J Neuroradiol 2002; 23: 671-673.

7. Marien A, Maris M, Verbeke S, et al. An unusual tumour causing neonatal respiratory distress. B-ENT 2012; 8 :149-151.

8. Sokolov M, Jecker P, Roth Y. Nasal teeth associated with rhinosinusitis. Rhinology 2004; 42: 167-170.

9. Lee FP. Endoscopic extraction of an intranasal tooth: a review of 13 cases. Laryngoscope 2001; 111: 10271031. 